\title{
Organizaciones indígenas de Latinoamérica y prácticas de comunicación para el desarrollo en el presente siglo
}

\author{
Eliza Carolina Vayas Ruiz \\ Universidad Técnica de Ambato, Ecuador \\ elivayasr@uta.edu.ec \\ Álvaro Jiménez Sánchez \\ Universidad Técnica de Ambato, Ecuador \\ al.jimenez@uta.edu.ec
}

\author{
Nelly Guamán Guadalima \\ Universidad Técnica de Ambato, Ecuador \\ ng.guaman@uta.edu.ec \\ Teresa Paredes Ruíz \\ Universidad Técnica de Ambato, Ecuador \\ tdi.paredes@uta.edu.ec
}

Resumen. El presente estudio sistematiza y describe las prácticas y tendencias de comunicación para el desarrollo llevadas a cabo por organizaciones indígenas de varios países latinoamericanos, como la producción de los medios de comunicación con carácter comunitario, las plataformas virtuales y la creación de diversos espacios de participación. Estas prácticas se configuran en un escenario complejo, donde converge la pobreza, la desigualdad social, las brechas digitales, las contradicciones ideológicas y el posicionamiento acelerado de la industria cultural occidental, que se confronta con la multiculturalidad y con la memoria histórico-cultural de la región. Se reflexiona sobre el papel de internet en las sociedades originarias, la cual se asume como un recurso valioso para la creación de plataformas infocomunicativas que alientan procesos de cambio mediante la producción de contenidos, la interacción y la emancipación ciudadana. Nos apoyamos en la revisión bibliográfica de fuentes documentales y de sitios web.

Palabras clave: comunicación / indígenas / desarrollo / cambio social / medios comunitarios 


\title{
Indigenous organizations in Latin America and communication practices for development in the current century
}

\begin{abstract}
Aвstract. The present study systematizes and describes the communication practices and trends for development carried out by indigenous organizations from several Latin American countries, such as community mass media production, virtual platforms, and the creation of different spaces for participation. These practices form in a complex scenario, where poverty, social inequality, digital gaps, ideological contradictions and the accelerated positioning of the western cultural industry converge. Such scenario is confronted with multiculturalism and the historical-cultural memory of the region. The study reflects on the role of the Internet in original societies, which is assumed as a valuable resource for the creation of info-communication platforms that encourage processes of change through the production of content, interaction and citizen emancipation. We rely on the bibliographic review of documentary sources and websites.

Keywords: communication / natives / development / social change / community media
\end{abstract}




\section{Introducción}

$\mathrm{D}$ espués de la Segunda Guerra Mundial, se iniciaron exploraciones teóricas que intentaron esclarecer cómo la comunicación puede ser utilizada como herramienta valiosa para promover procesos de desarrollo. Asimismo, desde la segunda mitad de la pasada centuria, emergieron varios modelos teóricos que nacieron como consecuencia de las coyunturas nacionales; se destacaron las luchas contra el colonialismo y las dictaduras que tuvieron lugar en numerosos pueblos latinoamericanos. Figuran, los modelos liberal-causal, el marxista-socialista y el monístico emancipatorio. En este sentido, las categorías comunicación popular y alternativa emergen en la década de los ochenta.

Las teorías que aparecieron en los años ochenta y noventa convergieron y dieron paso a un enfoque más integrador que dispone de lo cultural y de la participación ciudadana como dimensiones cardinales para el desarrollo. Igualmente, durante los últimos años, estas perspectivas se encuentran en un proceso de enriquecimiento epistemológico a partir de las nuevas tendencias comunicativas que emergen en el continente, particularmente en sectores comunitarios, indígenas, sindicales, grupos juveniles, feministas, etcétera. Sin embargo, la revisión realizada por los autores demuestra que las investigaciones o publicaciones que examinan las prácticas comunicativas para el desarrollo de los pueblos originarios son insuficientes, máxime cuando comprender la multiculturalidad que se da en estos fenómenos supone un reto reconocible.

Las transformaciones sociales que son impulsadas por los pueblos indígenas latinoamericanos en pleno siglo xxI son complejas y profundas, y en ellas figura la puesta en marcha de estrategias, políticas y proyectos encaminados a generar cambios sociales mediante prácticas de comunicación alternativa, emergente, popular, democrática y participativa. Sin embargo, "los avances aún no son suficientes porque no es fácil revertir extensos procesos históricos de opresión y sometimiento" (Rodríguez-Mir, 2008, p. 16).

Sobre estas temáticas se reflexionará en el presente artículo, el cual pretende dar respuesta someramente a las siguientes interrogantes: ¿qué aspectos generales distinguen a las sociedades y organizaciones indígenas latinoamericanas durante los últimos años? ¿Cuáles son sus principales prácticas de comunicación para el desarrollo y qué rasgos comparten en común?

Para ello, se examinan las principales problemáticas económicas, políticas, sociales y culturales que afrontan sus habitantes, se ejemplifican los movimientos sociales y populares indígenas de la región y se alude a las causas que han fortalecido sus luchas en los últimos años. Se aborda sintéticamente los efectos tienen las brechas digitales y el silenciamiento de las culturas ancestrales y se reflexiona sobre la importancia de los medios indígenas y el uso de 
internet como soportes dinamizadores del desarrollo y en la preservación de la memoria histórico-cultural.

Este un artículo de reflexión y se apoya en la revisión bibliográfica documental y virtual de fuentes de referencia que abordan aspectos e informaciones medulares sobre los pueblos originarios, sus características, movimientos sociales, organizaciones, luchas y prácticas de comunicación para el desarrollo que realizan.

\section{Pueblos originarios de Latinoamérica}

Los pueblos originarios son aquellos cuyos habitantes comparten rasgos físicos, sociales y culturales en un mismo territorio donde han coexistido históricamente con antelación a los procesos migratorios. Con el término originario también "se indica que una persona pertenece a un lugar, donde ha nacido y donde siempre ha vivido" (GrégorBarié, 2013, p. 61). Anterior a la llegada de los españoles al continente americano, estas culturas tenían una forma de convivir en sociedad en plena armonía con la naturaleza, lo cual se refleja en sus tradiciones, costumbres y prácticas sociales, culturales y comunicativas. Muchas de estas últimas servían como mecanismo para expresar sus herencias históricas e identitarias, al tiempo que formaban parte de su existencia.

Al hablar de culturas originarias, pensamos en los países de la región latinoamericana que aún cuentan con el privilegio de conservar la multiculturalidad que aportan estos pueblos. Piénsese en Chile, Brasil, Ecuador, Colombia, Bolivia, Guatemala, Argentina, Paraguay, Uruguay, México, Perú, El Salvador, entre otros.

En una consulta realizada para la investigación Latinoamérica indígena en el siglo XXI, del Banco Mundial (2017), se revela que 42 millones de habitantes de Latinoamérica son indígenas, lo que representa el $8 \%$ del total de la población. México, Guatemala, Perú y Bolivia son los países que tienen las poblaciones más extensas, con más del $80 \%$ del total de la región; es decir, 34 millones de personas.

Actualmente, existen en Latinoamérica 522 pueblos indígenas que ocupan grandes extensiones geográficas del continente: el Chaco Ampliado, la Amazonía, la Orinoquia, los Andes, la Llanura Costera del Pacífico, el Caribe Continental, la Baja Centroamérica y la Mesoamérica. Es Brasil el que cuenta con mayor diversidad de culturas originarias, con un total de 241 comunidades (734 127 habitantes); le sigue Colombia, con 83 (1 392623 habitantes); México, con 67 (9 504184 habitantes), y Perú, con 43 (3 919314 habitantes). (Unicef, 2017).

Los censos de países como Bolivia, Guatemala y Ecuador dan cuenta de un elevado porcentaje de indígenas respecto a su población total: Bolivia con $71 \%$, Guatemala con 66 \% y Ecuador con 43 $\%$. Cada país tiene sus propias culturas y nacionalidades étnicas, aunque la falta de demarcación territorial y los flujos migratorios que acontecieron antes de la conquista y en sus inicios hicieron posible la expansión de múltiples etnias por Sudamérica y Centroamérica. 
En Chile, por ejemplo, se reconoce la presencia de ocho culturas originarias: los atacameños, los diaguitas, los chiquillanes, los mapuches, los pehuenches, los tehuelches, los chonos y los alacalufes.

Pese a las imprecisiones que se han reflejado en algunos censos, en Ecuador se habla de trece nacionalidades indígenas, entre ellas la kichwa, la shuar, la achuar, la chachi, la epera, la huaorani, la siona, la secoya, la awa, la tsáchila, la cofán y la zápara. Asimismo, se reconocen como pueblos o grupos étnicos aquellos que salvaguardan su identidad de acuerdo a las tradiciones, el lenguaje, el espacio geográfico y las actividades económicas. Menciónense, por ejemplo, a los cañaris, los caranquis, los cayambis, los chibuleos, los guarangas, los natabuelas, los otavalos, los panzaleos, los puruhás, los quisapinchas, los salasacas, los saraguros y otros. A estos también se les conoce como pueblos amerindios, herederos de antiquísimas culturas preincaicas cuya lengua es el quechua.

Muchas de las características de los pueblos originarios están estrechamente relacionadas al contexto geográfico donde se construyen sus comunidades. Lo territorial ha constituido siempre un elemento de importancia vital para los miembros de la colectividad, donde se producen y reproducen sus relaciones históricas y cotidianas, y donde se edifica la identidad.

Los indígenas, desde sus inicios como comunidades originarias, mantienen un vínculo especial con la tierra y con su territorio, del cual dependen para su supervivencia, su organización y su socialización. Las prácticas de apropiación y explotación que se dieron durante la Colonia provocando que este vínculo se afianzara aún más.

Para ellos, todo cuanto les rodea está vivo $\mathrm{y}$, por tanto, la tierra es sagrada y divina. La convivencia en armonía, los saberes ancestrales que conectan sus prácticas con el cosmos y con las deidades veneradas durante siglos, las creencias, la literatura, las tradiciones, los instrumentos musicales de elaboración propia, los rituales, los mitos, las artesanías propias, la orfebrería, los alimentos, los remedios, las denominaciones que dan a todo cuanto les rodea - como el paisaje, las montañas, los ríos y lagos, los animales y las plantas, etcétera- constituyen un caleidoscopio de prácticas y formas simbólicas con profundas raíces históricas. Por el hecho de contar con culturas ancestrales diversas, a estos países se los considera como multiétnicos, plurinacionales y pluriculturales.

Con origen en la lengua quechua, el término pachamama que significa 'madre tierra', en países como Bolivia, Argentina, Ecuador, Chile y Perú, adquiere una connotación simbólica superior ya que se refiere a la representación de la tierra y de la naturaleza en toda su amplitud, y la interacción directa de esta con los seres humanos.

En honor a las deidades veneradas, como el sol y la madre tierra, se efectúan prácticas culturales representativas, como el Inti Raymi o Fiesta del Sol, que 
se desarrolla en numerosos pueblos indígenas latinoamericanos, como Ecuador, como apertura a la época de cosecha.

La economía de subsistencia, la organización comunal, el trabajo colectivo o minga, los cargos establecidos y los sistemas normativos propios son también elementos estructurales de estos pueblos. De igual forma, un concepto clave para entender la estructuración de las sociedades indígenas enmarcadas fundamentalmente en centros periféricos/rurales es el de autodesarrollo comunitario. Se trata de una perspectiva que implica, para los habitantes, una concepción propia de desarrollo, de lo que necesitan para subsistir y convivir en condiciones favorables y armónicas, así como la convergencia entre las voluntades y los recursos disponibles a su alcance para generar cambios en función de los intereses colectivos.

Los elementos mencionados como rasgos distintivos de los pueblos originarios han estado siempre determinados por prácticas de resistencia, armonía, convivencia y solidaridad; son parte indiscutible de la multiculturalidad, no solo de sus entornos comunitarios y nacionales, sino también de las peculiaridades culturales distintivas de la región latinoamericana y caribeña. Pero debe considerarse lo siguiente:

La identidad indígena no implica el ser idénticos los unos a los otros, es más bien una celebración de la diferencia, de la llamada diversidad, justamente porque una gran variedad de grupos indígenas convive dentro de un mismo Estado. Cada pueblo indígena posee diferentes y específicas normas, valores, símbolos y prácticas culturales. Sin embargo, se encuentran unidos por una historia común de dominación, marginalización y luchas reivindicativas, lo que fortalece la unidad en la diversidad de los pueblos indígenas. (Ayriwa-Pilco, 2000, párr. 80)

\section{Situación de los pueblos indígenas}

Los desafíos económicos, políticos, sociales y culturales que han afrontado los pueblos latinoamericanos durante décadas son complejos y han tenido una repercusión directa en sus culturas ancestrales, sobre todo en la actualidad. La pobreza, la desigualdad, la marginación, la insalubridad, las epidemias, la exclusión y la desintegración afectan diariamente su existencia. Desde el punto de vista laboral, prima el desempleo, el trabajo en condiciones precarias y la injusta remuneración. La situación es mucho más crítica para el sector femenino, víctima de brechas salariales respecto del masculino.

Cuando se revisan algunas plantillas de plazas de centros laborales, fábricas e industrias, es posible percatarse de que, en no pocos lugares, si se es indígena, no se cobra lo mismo que los ciudadanos que no lo son, aunque se trate de los mismos puestos y de similares niveles de escolaridad.

Diversas fuentes arrojan datos sobre el estado crítico en que se encuentran los pueblos originarios latinoamericanos. Más de 220 millones de personas de la región viven en la pobreza, y de 
estos, aproximadamente la mitad, en condiciones extremas (Programa de las Naciones Unidas para el Desarrollo [PNUD], 2016).

Políticas y leyes que promueven y garantizan el respeto a las culturas originarias, asícomolas queapoyanel progreso social de las comunidades marginadas son vulneradas. La explotación desmesurada de los recursos naturales - como el petróleo y la madera- en territorios donde radican estas poblaciones, el poco respeto hacia los procedimientos y regulaciones internacionales y nacionales para el uso responsable de los recursos naturales, así como la usurpación territorial para el establecimiento de industrias que favorecen el enriquecimiento de corporaciones transnacionales de países occidentales, constituyen otras causas que generan en estas comunidades descontento, rebeldía y resistencia.

Tampoco debe desestimarse la imposición de la globalización en la región latinoamericana y caribeña, que ha tenido efectos devastadores debido a la aplicación de prácticas neoliberales en los pueblos originarios. Se reconoce, por ejemplo, la privatización de las industrias nacionales y locales por corporaciones foráneas, la explotación desmesurada de los recursos naturales en territorios indígenas, el establecimiento de agencias y corporaciones mediáticas que favorecen la imposición de esquemas y modelos de la industria cultural occidental, etcétera.

Es cierto que la globalización favorece que los pueblos compartan sus economías, sus prácticas sociales y culturales; sin embargo, no hay que olvidar que también se encuentra inmersa dentro de una dimensión capitalista y neoliberal, ciertamente invasora, que puede eliminar el derecho de autonomía de los pueblos. El empeño por devastar la identidad y por implantar sistemas occidentales en la región hace posible un acelerado deterioro de la identidad cultural de estas comunidades.

El caminar de nuestros pueblos indígenas enfrenta hoy en día las políticas neoliberales que impulsan los grandes centros del capital transnacional a través de gobiernos títeres, puesto que dichas políticas pretenden el desmantelamiento de nuestras culturas milenarias, de nuestros saberes y el despojo descarnado de nuestras tierras, territorios y recursos naturales, acrecentando la pobreza y la migración de nuestras familias, así como la entrega de nuestras riquezas naturales a las grandes empresas transnacionales. (ZambranoCabanilla, 2006, párr. 15)

La migración, por su parte, es otro fenómeno que ha distinguido a las sociedades indígenas de la región. Desde hace siglos tienen lugar traslados de pobladores indígenas de sus territorios, tanto los que se dirigen hacia centros urbanos de un mismo contexto como los del tipo de migración contemporánea (el cruce de fronteras hacia otros países), causada fundamentalmente por las necesidades económicas y las influencias foráneas. Se añaden también los desplazamientos forzados, por cuestiones políticas, ambientales y de reunificación familiar. 
Llegar a esta situación se debe a múltiples factores, aunque lo que más ha influido es que las políticas nacionales respondan a intereses foráneos, sobre todo occidentales. "En general, los contextos políticos en América Latina favorecieron distintos modos de opresión, marginación y exclusión hacia los pueblos indígenas" (Rodríguez-Mir, 2008, p. 164).

\section{Movimientos sociales y populares indígenas}

La situación antes referida ha hecho que en las últimas décadas surjan movimientos sociales y populares indígenas que reclaman el reconocimiento de sus culturas originarias, sus derechos como ciudadanos, la erradicación de la discriminación, la violencia y la nacionalización de la riqueza en función del desarrollo económico de la colectividad.

Ha de reconocerse el quehacer de países como Bolivia, Venezuela y Ecuador, a propósito de las políticas públicas inclusivas que desde hace algunos años se han implementado para favorecer a los sectores indígenas, preteridos de la escena pública y desposeídos de derechos políticos, económicos y sociales durante siglos. Cabe indicar que "los movimientos indígenas están formados por las luchas por la identidad y la necesidad para abrir un espacio para la sobrevivencia dentro de la política nacional, y en ambiente económico y social" (Vargas-Hernández, 2005, p. 454).

Las organizaciones sociales y populares indígenas de Latinoamérica son numerosas y poseen diversas características. Según su alcance, pueden clasificarse en locales comunitarias, nacionales, regionales e internacionales.

En el siguiente listado pretendemos mostrar varias de estas organizaciones, las cuales se han destacado por generar sus propias prácticas comunicativas con fines de asociacionismo, participación, reconocimiento, emancipación y equidad. A tono con los beneficios de las TIC en los últimos años, la mayoría de ellas cuentan con medios comunitarios propios, con páginas web y sitios de redes sociales.

Tabla 1. Organizaciones y movimientos indígenas de Latinoamérica

Organización Amazónica de Pueblos Indígenas de Pastaza (OPIP)

Confederación de Nacionalidades Indígenas del Ecuador (CONAIE)

Asociación de Cabildos Indígenas del Norte del Cauca (ACIN)

Movimiento indígena aimara

Confederación de Pueblos Indígenas de Bolivia (CIDOB)

Coordinadora de las Organizaciones Indígenas de la Cuenca Amazónica (COICA)

(continúa) 
(continuación)

Grupo Internacional de Trabajo sobre Asuntos Indígenas (IWGIA, por sus siglas en inglés)

Movimiento de la Juventud Kuna, en Panamá

Asociación Interétnica de Desarrollo de la Selva Peruana (AIDESEP)

Unión de Organizaciones de la Sierra Juárez de Oaxaca (UNOSJO)

Organización Nacional de Pueblos Indígenas de Argentina (ONPIA)

Organización Nacional Indígena de Colombia (ONIC)

Alianza Estratégica Aymaras Sin Fronteras

Enlace Continental de Mujeres Indígenas (ECMIA)

Foro Internacional de Mujeres Indígenas (FIMI)

Alianza Internacional de los Pueblos Indígenas y Tribales de los Bosques Tropicales (IAITPTF, por sus siglas en inglés)

Foro Permanente para las Cuestiones Indígenas de la ONU (UNPFII, por sus siglas en inglés)

Asociación Indígena de la República Argentina (AIRA)

Centro Integral de Capacitación y Desarrollo Kallawaya (CICADEKA), en Bolivia

Asociación de Mapuches Urbanos (AD-MALEN Y KASAWAIÑ), en Chile

Asociación de Mapuches Urbanos Lelfünche (MAPUCHES URBANOS), en Santiago de Chile

Consejo Regional Indígena del Cauca (CRIC), en Colombia

Centro para el Desarrollo Indígena (CEDIN), en Costa Rica

Centro Cultural Abya Yala, en Ecuador

Consejo de Desarrollo de las Nacionalidades y Pueblos del Ecuador (CODENPE)

Confederación de Pueblos de la Nacionalidad Kichwa del Ecuador (ECUARUNARI)

Asociación Nacional de Indígenas Salvadoreños (ANIS)

Consejo Indígena Popular de Oaxaca “Ricardo Flores Magón” (CIPO-RFM)

Centro de Culturas Indígenas del Perú (CHIRAPAQ)

Consejo de la Nación Charrúa (CONACHA), en Uruguay

Consejo Nacional Indio de Venezuela (CONIVE)

Elaboración propia 
Varios movimientos con propósitos similares son los zapatistas civiles, en México; la movilización de los excluidos como los Sin Tierra, en Brasil y, los Sin Empleo, en Argentina. Cabe destacar que, si bien estas modalidades organizativas no comparten rasgos tradicionales de asociacionismo - con personalidad jurídica o afiliaciones sujetas a estructuras con domicilio legal y reglamentaciones-, su nivel de cohesión voluntaria, de unidad, comunión y participación las convierte simbólicamente en organizaciones sociales de reconocimiento. De esta forma "quienes fueron actores políticos marginales pasan a ocupar espacios en la escena política nacional y [...] se convierten en voceros para las decisiones nacionales sobre el desarrollo" (MacDonald, 2003, p. 3).

Hoy los movimientos sociales indígenas de Latinoamérica tienen una influencia superior a la de años anteriores; se han llegado a convertir en importantes estructuras políticas con incidencia en organismos internacionales de derechos humanos, gracias a su inserción en numerosas organizaciones no gubernamentales (ONG). También es notable cómo los procesos de globalización favorecieron el afianzamiento de los movimientos de los pueblos indígenas y sus luchas, pues se valieron de los grandes medios de comunicación para posicionar, en la esfera pública internacional, todo aquello que les afectaba y que constituía flagrantes violaciones de sus derechos y culturas.

Algunos de los eventos más importantes que se llevan a cabo en
Latinoamérica dedicados a los pueblos y nacionalidades indígenas son el Foro Internacional Indígena sobre Biodiversidad (FIIB), Encuentro Nacional de Pueblos Originarios (Argentina), Encuentro Multidisciplinario sobre Pueblos Indígenas (EMPI), el congreso internacional sobre interculturalidad organizado por el Centro Internacional de Estudios Superiores de Comunicación para América Latina (CIESPAL) y la Sociedad Latinoamericana de Estudios Interculturales (SOLEI), el Congreso Internacional de Pueblos Originarios, el Congreso Internacional América Latina e Interculturalidad, el Congreso Internacional Los Pueblos Indígenas de América Latina, y el Encuentro Internacional sobre Pueblos Indígenas, organizado por la Organización Panamericana de la Salud (OPS/OMS).

Auspiciados por las organizaciones indígenas, centros especializados, universidades latinoamericanas $y$ organismos internacionales, estos eventos se convierten en importantes espacios de socialización, discusión e intercambio para la puesta en marcha y divulgación de estrategias, campañas y proyectos locales, regionales y nacionales que pretenden acercarse a la realidad de estos pueblos, a sus luchas ancestrales, y que tienen como objetivo contribuir a preservar y fortalecer la memoria histórico-cultural.

\section{Brechas digitales e invisibilización indígena}

En los últimos años, se ha evidenciado un auge en el uso de las TIC por parte de 
las sociedades indígenas de la región latinoamericana y caribeña, a propósito de las políticas de acceso llevadas a cabo por varios países, como Venezuela, Bolivia, Ecuador, Argentina, Brasil, Nicaragua y Uruguay. Ahora, en muchos de estos contextos, las tecnologías se usan colectivamente desde centros educativos, organizaciones sociales, centros de internet, o con el modelo del café internet en algunas poblaciones rurales. Se añade el incremento de la telefonía móvil y la presencia en las redes sociales (YouTube, Facebook, WhatsApp, etcétera).

Sin embargo, pretendemos detenernos en cómo se ha demostrado, desde distintos estudios y plataformas, las desventajas quehan matizadoa este sector desde inicios del siglo xxI. Los registros de la corporación Google, por ejemplo, unidos a los estudios emprendidos desde centros de investigación y comunidades académicas de Iberoamérica, dan cuenta de la desigualdad y los limitantes que existen para que los sectores indígenas accedan a las tecnologías de la información y la comunicación (TIC).

Las investigaciones arrojan que los indígenas latinoamericanos son los más afectados en este sentido. Si bien se reconoce a la región como el mercado favorito para las grandes compañías comercializadoras de tecnologías, laptops, teléfonos celulares, tablets, etcétera, el acceso a ellos es altamente desigual en las distintas regiones geográficas y grupos sociales.
Esta desigualdad contribuye al aumento de la brecha entre aquellos que tienen acceso a abundantes fuentes de información y los que se ven desprovistos de ellas, lo que refuerza la marginación que ya existe en términos de desarrollo y de recursos técnicos.

En Bolivia, El Salvador, Guatemala, Honduras y Paraguay, solo el $5 \%$ del total de la población tiene acceso a internet, mientras que en Costa Rica, Chile, Brasil y Uruguay el acceso oscila entre el 19 y $30 \%$. Las brechas digitales en los pueblos indígenas cobran "un rostro particular y se hacen más profundas si se observa en detalle la situación de oportunidades de uso, acceso y aprovechamiento de la comunicación, información y conocimiento" (Iniciativa de Comunicación para el Desarrollo Sostenible [ICDS], 2011, p. 6).

En estos tiempos, ya no solo se trata de las tendencias discriminatorias y de exclusión social, política y económica reconocidas tradicionalmente; los pueblos originarios "también se sienten marginados por la tecnología, sufriendo siempre la desventaja de no tener acceso al territorio ciberespacial que han creado las nuevas tecnologías de información y comunicación"11 (Ayriwa-Pilco, 2000).

Se impone la necesidad de promover la participación de estos pueblos en estos espacios, propiciar el acceso y manejo de las TIC y contribuir a erradicar las brechas digitales existentes. "Caso

1 Pese a los avances en la región durante los últimos cinco años, esta situación continúa siendo un problema irresuelto para los países sudamericanos que cuentan con nacionalidades indígenas. 
contrario, las actuales condiciones de exclusión social, económica y cultural de estos pueblos se agravará en detrimento de su diversidad e identidad cultural" (Agurto y Mescco, 2012, p. 22).

El Sistema de Información de Tendencias Educativas en América Latina (SITEAL) figura entre las organizaciones que han señalado y criticado la exclusión digital que viven los pueblos indígenas: "a pesar de la relevancia del uso de internet para acceder al conocimiento en un mundo globalizado, su acceso en la región (América Latina) aún no se ha democratizado" (Paiba-Samamé, 2013, párr. 3). Pero cuando se habla de brechas digitales, no solo se trata de accesibilidad, sino también de efectos como la invisibilización de determinados sectores de las sociedades contemporáneas latinoamericanas en estos espacios.

La escasez de contenidos locales, la falta de conocimientos sobre el uso de internet y el precio de la conexión y los dispositivos son las principales causas de la brecha digital en Latinoamérica, donde seis de cada diez personas con cobertura no son usuarios de la red. (ABC Tecnología, 2016, párr. 1)

Ponce de León (2011, p. 145) expresa que, en países como Argentina, "los medios audiovisuales han ido estigmatizando al indígena en una imaginería reduccionista alejada de la realidad. Alimentando la exclusión de los indígenas en la sociedad". Lamentablemente, esta realidad es ampliamente reproducida en numerosos países latinoamericanos, donde no son escuchados, no se les otorgan derechos constitucionales que favorezcan el reconocimiento de sus luchas e identidad y son víctimas del silenciamiento en las estructuras políticas y en los medios de comunicación.

Esta situación se agrava cuando por los medios de comunicación tiende a calificarse a los sectores indígenas como amenaza para la seguridad nacional o, como bien debiera decirse, de los gobiernos y sistemas políticos oligárquicos y derechistas cuyos intereses se ven afectados cuando las luchas y reclamos de los movimientos sociales y populares indígenas se fortalecen y van adquiriendo voz y espacio en la escena pública regional e internacional, "y en consecuencia aplican políticas públicas contrarias a los derechos, demandas y reivindicaciones de las sociedades nativas o en el mejor de los casos políticas que ignoran a las sociedades amerindias (invisibilización)" (Rodríguez-Mir, 2008, p. 7).

Por ejemplo, cuando se trata del cubrimiento y la representatividad de las comunidades indígenas, la tendencia del sistema de comunicación de masas ha sido muy consistente: optan por ignorar del todo dichas comunidades haciéndolas invisibles, por amontonarlas en un proceso de homogeneización o por presentarlas como simples actores pasivos, víctimas de un sistema injusto. Ahora, cuando las comunidades toman en sus manos el asunto en cuestión, los medios hegemónicos por lo general presentan dichas situaciones como actos criminales enfatizando su tendencia a actuar por fuera de la ley -bloquear carreteras, ocupar tierras de manera "ilegal", etc.-. (Alfonso-Murillo, 2011, p. 157) 
También hay que añadir la producción de contenido estereotipado y discriminatorio que hace referencia errada y ridiculizante de los sectores indígenas, además de la invisibilización de su riqueza sociocultural. Otros conceptos que explican la complejidad de estas tendencias son el mercantilismo informativo, la concentración mediática y la oligarquía de la comunicación. En su conjunto, expresan que los grandes medios de comunicación masiva están sujetos a prácticas e intereses lucrativos y se encuentran monopolizados por los sectores de poder de potencias occidentales. Si bien es cierto que muchas de estas compañías tienen sede en Latinoamérica, no responden a las realidades de la región, puesto que las ocultan, se omite el valor de su memoria histórico-cultural, identitaria y multicultural.

Sustentado en Stavenhagen (1999), el antropólogo social Javier Rodríguez-Mir (2008) aseveró que esta condición actual de los pueblos originarios está asociada a dos factores principales:

1. La progresiva destrucción de su economía y modos de vida.

2. El negar que son plenos ciudadanos en igualdad de condiciones dentro de los estados modernos. Estos transformaron a los pueblos indígenas en ciudadanos "invisibles" dentro de sus propios países, extraños en sus propias tierras y no reconocidos de forma legal ni formal como entidades colectivas.

Informes del Observatorio de la Discriminación en Radio y Televisión muestran cómo "la presencia de los pueblos originarios en los medios de comunicación es escasa y que roza la invisibilización total" (8300 web, 2010, párr. 1). Por lo general "aparecen en los medios cuando hay un caso muy resonante [...] ligado a conflictos de tierras con terratenientes y empresas que quieren desalojarlos [...], no se contextualiza la temática con sus derechos ancestrales reconocidos constitucionalmente" (8300 web, 2010, párr. 1).

\section{Tendencias y prácticas de comunicación para el desarrollo en sectores indígenas}

En los inicios de 1980, teóricos como Ramiro Beltrán, Paulo Freire, Juan Díaz Bordenave, entre otros, sientan las bases de la comunicación horizontal, la cual difiere de las perspectivas verticales y unidireccionales de los modelos clásicos de Aristóteles y Laswell. Aquí se defiende una comunicación que necesita tres elementos clave para poder incidir en el desarrollo: acceso, diálogo y participación.

Si bien hubo grandes aportes que ayudaron a definir la comunicación para el desarrollo en años precedentes, su proceso redefinitorio fluctúa entre las décadas de 1980 y 1990 del pasado siglo, cuyas postulaciones favorecen el valor de la participación social-ciudadana en los procesos de desarrollo. Asimismo,

la comunicación para el cambio social es el paradigma más reciente entre los descritos. De alguna manera ha estado 
siempre presente, en parte en las experiencias de comunicación alternativa y participativa, y en parte en las acciones de comunicación para el desarrollo. (Gumucio-Dagron, 2011, p. 19)

A tono con las circunstancias de la región latinoamericana, su historia y peculiaridades, surgen varios modelos de comunicación para el desarrollo, entre ellos el liberal-causal, el marxistasocialista y el monístico emancipatorio.

El modelo liberal-causal se refiere a la relación de causa-efecto y explica las mediaciones presentes en la producción social de la información y su relación con las particularidades económicas, políticas y sociales. El modelo marxista-socialista explica las relaciones que se establecen entre la comunicación y los procesos de desarrollo en su amplitud conceptual:

Sustenta en la difusión de la ideología y la potenciación de la conciencia nacional como elementos indispensables para el cambio social. Le atribuye valor agregado a la comunicación pública, como útil en la preservación de la independencia y la soberanía de los pueblos; defiende lo popular, la participación y la democracia (Mena y Álvarez, 2015, p. 210)

El modelo monístico emancipatorio realiza un examen de cómo los medios de comunicación y el desarrollo científico técnico poseen valor agregado. Se ocupa en visualizar y reflexionar en clave crítica acerca de los principales problemas sociales que afrontan los pueblos, y "fomenta y promueve la participación social como indispensable en los proyectos de desarrollo" (Mena y Álvarez, 2015, p. 210).
Hablar de comunicación para el desarrollo y el cambio social en Latinoamérica, particularmente en los pueblos indígenas, requiere entender cuan vulneradas se encuentran actualmente estas culturas y cuáles son los procesos que se vienen dando hace siglos. Estas realidades dan cauce a prácticas de resistencia y de reafirmación, a protestas y a reivindicaciones, para las cuales se recurre a los medios de comunicación masivos que resultan, asimismo, en otros movimientos de carácter comunitario, alternativo y contrahegemónico. A estos hay que añadir los proyectos, las políticas, las estrategias y las leyes de comunicación, en las que "el reto de la comunicación es fortalecer a la población para exigir sus derechos" (ICDS, 2010, p. 12).

Históricamente, la comunicación con perspectiva de desarrollo, proveniente de los pueblos indígenas del continente, se ha visto matizada por mediaciones económicas como un reflejo claro de lo que ha costado y siguen costando las nociones de desarrollo occidental (industrias extractivas como las minas, monocultivos industriales de maíz, papa y demás alimentos ancestrales que siempre se han cultivado en comunidad $\mathrm{y}$ en conjunto con otras producciones, etcétera). Sin embargo, la constitución de organizaciones, medios públicos, centros educativos y plataformas que, gracias a la tecnología, han propiciado nuevas interpretaciones en estos contextos, donde comienzan a entenderse otras dimensiones como parte de la vida en sociedad y del progreso humano; a saber, el derecho 
a la participación, la educación, el afianzamiento y el resguardo de sus culturas ancestrales y demás componentes que conforman la memoria histórica. Esto hace que se redefinan las nociones tradicionales de desarrollo que han existido y se han reproducido en diversos espacios mediáticos y de instrucción.

Hablar de comunicación es hablar de culturas y si hablamos de culturas, es hablar de diferencias de relaciones, de percepciones y del habla [...]. No se puede comprender la comunicación sin comprender los procesos históricos, sociales, económicos, políticos y culturales, toda vez que este componente atraviesa toda relación y construcción social. En esta diversidad cultural se inscriben los pueblos y nacionalidades indígenas como sociedades diversas. (Chuji-Gualinga, 2007, párr. 4).

Durante el Foro Permanente de las Naciones Unidas para las Cuestiones
Indígenas (UNPFII, por sus siglas en inglés)" ${ }^{2}$ cuyo lema era "Pueblos indígenas y comunicación para el desarrollo hacia un manejo sostenible de los recursos naturales y adaptación al cambio climático", se reconoció la importancia de la elaboración de una plataforma de comunicación "como un espacio de promoción al derecho a la comunicación de los pueblos indígenas" (ICDS, 2011, p. 22).

En Perú, por ejemplo, se destaca la Asociación Pukllasunchis, que surgió en 1992 y desde su génesis ha propiciado espacios de formación y capacitación, tanto de docentes como de comunicadores sociales. Entre sus temáticas prioritarias figuran la multiculturalidad y la interculturalidad, la educación intercultural bilingüe y la formación para la educación y la comunicación intercultural. Para dar cumplimiento a sus objetivos, esta organización fundó en el 2013 el Instituto Superior Pedagógico Privado Pukllasunchis.

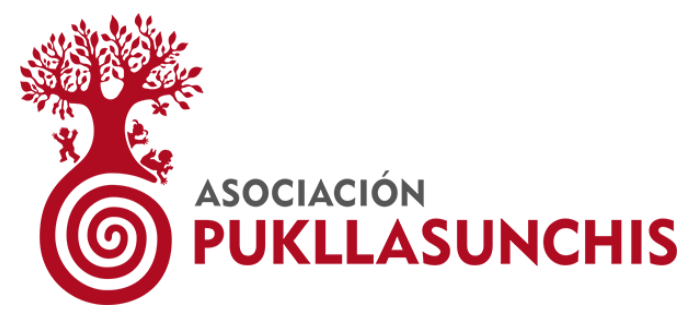

Fuente: Asociación Pukllasunchis, s. f.

2 Muchas de estas experiencias y propuestas fueron publicadas en un texto de sistematización de experiencias de comunicación de los pueblos indígenas de Latinoamérica, y se otorgó especial prioridad al manejo de recursos naturales y cambio climático. 
$\mathrm{Si}$ bien es cierto que el acceso a la tecnología está presente en algunos sectores indígenas de países latinoamericanos - aunque muy limitadamente-, en las culturas indígenas aún prevalece la oralidad y otras formas simbólicas sin mediación tecnológica, por lo que los proyectos de educación y comunicación para el desarrollo deben otorgar importancia a tales formas de interacción social. "De ahí que las estrategias que no incorporen estas formas tradicionales de comunicarse tienden a fracasar" (ICDS, 2011, p. 74).

Corresponde a los gobiernos nacionales, a sus ministerios de educación, de cultura, a las comunidades académicas, a las organizaciones y centros de investigación, a los medios de comunicación pública del escenario latinoamericano actual y a los propios actores sociales de las comunidades favorecer la integración para la creación de nuevas políticas de comunicación, campañas de bien público, leyes inclusivas de comunicación y proyectos de comunicación para el desarrollo, en los que se favorezca la formación y el fortalecimiento de capacidades en estos temas.

Otras instituciones y centros latinoamericanos que también han implementado importantes proyectos de comunicación para el desarrollo en sectores indígenas, que cuentan con sus plataformas virtuales y sitios de redes sociales son los de la tabla siguiente.

Tabla 2. Instituciones y centros latinoamericanos que han implementado proyectos de comunicación para el desarrollo en sectores indígenas

\begin{tabular}{l}
\hline Centro de Educación y Comunicación para los Pueblos Indígenas (CECOPI) \\
\hline Centro Andino de Acción Popular \\
\hline Centro de Culturas Indígenas del Perú (Chirapaq) \\
\hline Consejo Guatemalteco de Comunicación Comunitaria* \\
Centro de Formación y Realización Cinematográfica / Consejo Audiovisual Indígena de Bolivia \\
(CEEREC / CAlB) \\
\hline Centro Iberoamericano de Estudios en Comunicación, Información y Desarrollo (CIECID) \\
\hline Voces Nuestras. Centro de Comunicación \\
\hline Centro de Comunicación para el Desarrollo (CECODE), en Guatemala \\
\hline Centro Latinoamericano para el Desarrollo y la Comunicación Participativa (CDESCO) \\
\hline Red de Comunicación Indígena (RCI), en Argentina \\
\hline Red Centroamericana de Radios Comunitarias Indígenas \\
\hline Fundación Comunicándonos \\
\hline Programa Regional de Participación Política Indígena (PPI)** \\
\hline Universidad Autónoma Miguel de Cervantes \\
* Agrupa cinco asociaciones de radios comunitarias. \\
** Es patrocinado por la Fundación Konrad Adenauer. \\
Elaboración propia
\end{tabular}


Para las comunidades indígenas,

la actividad de la comunicación es una interacción continua, un permanente intercambio de información entre los muchos individuos que componen las comunidades locales, que resulta en una lógica colectiva, la cual, en último término, impulsa y dirige sus actos unificados. (Alfonso-Murillo, 2011, p. 163)

Desde el punto de vista académico y empírico, numerosas universidades latinoamericanas que cuentan con carreras de Comunicación Social se favorecen del desarrollo de investigaciones de grado y posgrado, así como del establecimiento de líneas de estudio, programas y asignaturas de comunicación para el desarrollo, con lo que se intenta otorgar legitimidad, integralidad y amplitud a un campo que se encuentra en proceso de crecimiento epistemológico.

El Observatorio del Derecho a la Comunicación de los Pueblos Indígenas “es una plataforma de documentación y seguimientodehechos, políticaspúblicas, legislaciones y procesos relativos al Derecho de los Pueblos Indígenas" (Coordinadora Latinoamericana de Cine y Comunicación de los Pueblos Indígenas, s. f.). Asimismo, la Plataforma de Comunicación y Desarrollo de los Pueblos Indígenas de América Latina se describe de la siguiente forma:

Es un espacio de participación que procura incidir en las políticas de comunicación y desarrollo de los pueblos indígenas, mediante mecanismos e instrumentos que permitan concertar prioridades y fortalecer las capacidades en función de objetivos de desarrollo, autodeterminación y gestión de territorios indígenas [...] constituye un medio para el intercambio de conocimientos, propuestas y mecanismos para la articulación y la cooperación entre diferentes actores comprometidos con los pueblos indígenas. (La Iniciativa de Red de Comunicación, 2009, párr. 1)

El Centro de Mujeres Comunicadoras Mayas de Guatemala cuenta con su propia página web, Mirador de Desarrollo de Guatemala, plataforma desde la cual "cuentan y muestran al mundo todas las experiencias del trabajo comunitario y la utilización de las nuevas tecnologías de comunicación e información para los intereses de los pueblos indígenas dentro y fuera del país" (Ayriwa-Pilco, 2000).

El Centro Internacional de Información y Documentación de los Pueblos Indígenas (CIIDPI) es una iniciativa gestada durante el Primer Encuentro de Periodistas Indígenas que organizó Naciones Unidas a finales de enero de 1998 en Madrid, España.

Numerosos seminarios, foros $y$ eventos han apoyado el diálogo multicultural con el objetivo de afianzar el respeto hacia las identidades mediante la creación de proyectos comunitarios y la búsqueda de soluciones a las problemáticas que afrontan los pueblos originarios. Baste mencionar el Congreso Nacional de Comunicación Indígena, en México; la Cumbre Continental de Comunicación Indígena, en Colombia; el Encuentro Internacional en Comunicación para el Desarrollo de los Pueblos Indígenas de América Latina, y el Seminario Pueblos Indígenas, 
Medios de Comunicación y Televisión Digital, auspiciado por la Secretaría de Telecomunicaciones de Chile, con apoyo de la Embajada de Canadá y el Programa de las Naciones Unidas para el Desarrollo (PNUD).

Estos y otros espacios de intercambio y socialización han facilitado que los pueblos originarios latinoamericanos implementen declaraciones y pronunciamientos sobre comunicación, que entre sus temas de interés indican las brechas digitales, el derecho a la comunicación y la libre expresión, los medios indígenas y su papel en los procesos de transformación social y emancipación, la lucha contra la censura, el periodismo ciudadano y el reclamo por políticas y leyes inclusivas de comunicación.

La tabla 3 expone los pronunciamientos y las declaraciones vinculadas a la comunicación social de los pueblos indígenas latinoamericanos. Se demuestra cómo, en su mayoría, se efectuaron durante la primera década del presente siglo, años en los cuales las brechas digitales y el derecho a los espacios de participación fueron temas constantes en las agendas públicas de numerosos eventos efectuados en el continente.

Tabla 3. Pronunciamiento y declaraciones de los pueblos indígenas latinoamericanos vinculados a la comunicación.

\begin{tabular}{lll}
\hline Pronunciamiento / Declaraciones & País & Año \\
\hline $\begin{array}{l}\text { Declaración de los Pueblos Indígenas ante la Cumbre Mundial de la } \\
\text { Sociedad de la Información }\end{array}$ & Suiza & 2003 \\
\hline $\begin{array}{l}\text { Segunda Cumbre Continental de Pueblos y Nacionalidades Indígenas de } \\
\text { Abya Yala }\end{array}$ & Ecuador & 2004 \\
\hline $\begin{array}{l}\text { Declaración de Misak Wampia en el Primer Encuentro de Comunicación } \\
\text { Indígena }\end{array}$ & Colombia & 2006 \\
\hline $\begin{array}{l}\text { Comunicación y Pueblos Indígenas: Asumiendo el Desafío Tecnológico } \\
\text { Declaración del I Congreso Nacional de Comunicación Indígena de } \\
\text { México }\end{array}$ & México & 2006 \\
\hline $\begin{array}{l}\text { Declaración del IX Festival Internacional de Cine y Video de los Pueblos } \\
\text { Indígenas }\end{array}$ & Bolivia & 2008 \\
\hline $\begin{array}{l}\text { Tercera Cumbre Continental de Pueblos Indígenas } \\
\text { Declaración de los Comunicadores Indígenas del Perú en el Segundo } \\
\text { Encuentro Nacional de Comunicadores Indígenas del Perú. }\end{array}$ & Gerú & 2008 \\
\hline $\begin{array}{l}\text { Declaración del Segundo Congreso Nacional de Comunicación Indígena } \\
\text { (CNCl) }\end{array}$ & México & 2008 \\
\hline $\begin{array}{l}\text { Desafío tecnológico y marco legal para una comunicación plurinacional. } \\
\text { Documento final de conclusiones del Quinto Taller Internacional de } \\
\text { Comunicación Indígena }\end{array}$ & Ecuador & 2009 \\
\hline $\begin{array}{l}\text { Pronunciamiento Político del III Congreso Nacional de Comunicación } \\
\text { Indígena }\end{array}$ & México & 2010 \\
\hline $\begin{array}{l}\text { Declaración de la Cumbre Continental de Comunicación Indígena de } \\
\text { Abya Yala }\end{array}$ & Colombia & 2010 \\
\hline $\begin{array}{l}\text { Declaración de Ajalpan en el IV Congreso Nacional de Comunicación } \\
\text { Indígena }\end{array}$ & México & 2011 \\
\hline
\end{tabular}

Elaboración propia 
Los denominados medios comunitarios se constituyen como impulsores efectivos para generar audiencia, participación ciudadana y favorecer procesos de desarrollo por medio de la tecnología.

Son vitales para visualizar este gran avance hacia sus propias comunidades y al exterior. Contemplar los medios como soportes reales que ayuden a reforzar la reconstrucción de la identidad indígena y de la ciudadanía, además de ayudar a la interacción con otros grupos de la sociedad urbana es también parte de la labor. (Chaparro-Escudero, 2003, p. 165)

Las radios mineras bolivianas ${ }^{3}$ son consideradas pioneras en la comunicación participativa, popular, democrática y alternativa. Se destacan Radio Nacional de Huanuni (Huanuni, Oruro), Radio Vanguardia (Colquiri, La Paz) y Radio 16 de Marzo (Bolívar, Oruro). Asimismo, se reconoce en Colombia a Radio Sutatenza, además de otras experiencias similares en Brasil, Perú y Chile. Otros ejemplos tienen lugar en México, donde se destacan las radios vía satélite, como La Hora Mixteca, en la ciudad de Fresno, California, que se trasmite en mixteco desde California hasta Oaxaca y en otras partes del país.

Dicha producción de comunicación pública en Latinoamérica demuestra que estamos en presencia de formas genuinas de autodesarrollo comunitario, en las que los protagonistas son los habitantes de estos contextos y las agendas responden a sus problemáticas y a sus intereses particulares. De este modo, se expresa un desplazamiento de la concepción sujetos sociales a actores sociales legítimos y activos.

Según Beltrán, durante mucho tiempo, estos medios se autofinanciaban y su producción se distinguía por ser "no partidaria, autogestionaria, sin publicidad comercial y practicando verdaderamente la democracia en la comunicación" (1993, p. 23). También asevera que los mineros bolivianos se fueron estableciendo como los antecesores de la comunicación alternativa antes de que estudiosos implantaran las bases teóricas.

Herrera-Miller afirma que la praxis comunicativa minera se constituye como un espectro muy rico y amplio "para indagar sobre los procesos no sólo de comunicación popular o alternativa, sino también de las relaciones entre comunicación, desarrollo, cambio social, política, movimientos sociales, participación, diálogo, entre otros" (2005, p. 53).

Otros medios destacados son las radios cristianas en Colombia y Chile, los altoparlantes en los barrios populares de Lima y las tiras cómicas en Buenos Aires y Ciudad de México. Por

3 La comparación entre las fechas de fundación y producción de muchos de estos medios demuestra cómo se cumple lo formulado por Ramiro Beltrán, en varios de sus estudios, al destacar que la práctica antecedió a la teoría en este campo. 
su parte, han sido los grupos feministas, los asambleístas y los jóvenes indígenas de Brasil, México y Nicaragua quienes más han destacado por el uso de internet.

Ha sido importante el quehacer de la Red de Radios Públicas, Privadas y Comunitarias de Ecuador, integrada por trece radios de trece nacionalidades indígenas del país desde el 2009, y ha de reconocerse la crisis e inestabilidad que han afrontado por falta de financiamiento a causa de la supeditación institucional en muchos casos.

Perú cuenta con numerosos medios representativos de este sector. Se destaca Radio Wampis, medio de comunicación indígena que se proclama en defensa de la Amazonía y sus pueblos. Se ubica en
Soledad, comunidad nativa enmarcada en el distrito Río Santiago, provincia de Condorcanqui, en la región Amazonas.

Asimismo, significativa ha sido la Ley de Radios Comunitarias de Guatemala, orientada a democratizar la libertad de expresión y a favorecer que las comunidades indígenas cuenten con espacios para recibir información, participar $y$ divulgar sus culturas ancestrales.

Es justo mencionar el periódico de los pueblos indígenas Wayuunaiki, primer medio alternativo, étnico y comunitario de Venezuela y del Departamento de La Guajira (Colombia). En este último país también funciona un conjunto de emisoras radiales indígenas que se enmarcan en distintas partes del territorio nacional, como se muestra en la tabla 4.

Tabla 4. Emisoras radiales indígenas de Colombia

\begin{tabular}{|c|c|c|}
\hline Nombre de la emisora & Pueblo indígena & Localización \\
\hline Namui Wan Estéreo & \multirow[t]{3}{*}{ Misak } & Zona Oriente, Municipio de Silvia \\
\hline Uswal Nasa Yuwe & & Zona Nororiente, Municipio de Caldono \\
\hline $\begin{array}{l}\text { Tejido Tel Yuwe: (Nuestra Voz } \\
\text { Estéreo, Radio Identidad- } \\
\text { Chimborazo, Programa de Jóvenes) }\end{array}$ & & Zona Occidente, Municipio de Morales \\
\hline Voces de Nuestra Tierra de Jambalo & \multirow{5}{*}{ Nasa } & Zona Norte, Municipio de Jambalo \\
\hline Nasa Estéreo & & Zona Norte, Municipio de Toribio \\
\hline Emisora Canoas Estéreo & & Zona Norte, Municipio de Caloto \\
\hline Radio Payumat & & Zona Norte, Municipio de Santader \\
\hline Radio Nasa de Tierradentro & & $\begin{array}{l}\text { Zona Tierradentro, Municipio de } \\
\text { Belalcázar }\end{array}$ \\
\hline Renacer Kokonuco & Kokonuco & Zona Centro, Municipio de Puracé \\
\hline Aires del Pueblo Yanakona - Stereo & Yanakona & Zona Sur, Municipio de la Sierra \\
\hline $\begin{array}{l}\text { Programa de Comunicación y } \\
\text { Cultura - Radio Libertad }\end{array}$ & Totoró & Zona Oriente, Municipio de Totoró \\
\hline
\end{tabular}

Fuente: Consejo Regional Indígena del Cauca, s. f. 
La perspectiva de desarrollo en estos medios de comunicación se visualiza a partir del criterio de que se convierten en importantes "Centros de Comunicación Popular que constituyen plataformas de formación en el uso de los medios locales de comunicación, de producción audiovisual de contenidos que apoyen acciones vecinales, campañas en pro de una mejora en la calidad de vida" (Chaparro-Escudero, 2003, p. 169).

La llegada del siglo xxi ha estado asistida por un conjunto de cambios políticos, económicos, sociales y culturales a escala global, en el que el progreso tecnológico favorece nuevas formas de producción simbólica por medio del ciberespacio e impacta vertiginosamente en el establecimiento de las relaciones cotidianas de los sujetos sociales.

$\mathrm{Si}$ bien se reconocen las brechas digitales referidas anteriormente, es evidente que, en plataformas virtuales, páginas web y sitios de redes sociales como Facebook, Twitter e Instagram, se han incrementado los contenidos que abordan cuestiones importantes de las culturas ancestrales latinoamericanas.

Una dificultad aún presente, en cuanto al uso de la plataforma de internet para producir contenido de interés de las culturas indígenas de varios países latinoamericanos, es la ausencia de estos en el proceso de creación de las páginas y la necesidad de participación ciudadana en la producción de contenido. En muchas ocasiones son espacios administrados por organizaciones civiles, ONG, universidades, centros de estudio y personas independientes, pero no insertan en los proyectos a quienes han de considerarse actores clave de las iniciativas. "La participación de los actores involucrados es esencial en las propuestas de comunicación para el desarrollo, alternativa y participativa, que son las expresiones más reconocidas de la comunicación para el cambio social" (Gumucio-Dagron, 2011, p. 28).

Jesús González-Pazos señala dos elementos importantes en lo referente a la comunicación para el desarrollo en comunidades indígenas: el proceso de retroalimentación y la aculturación. Es en este sentido que los agentes externos han de tomar conciencia de las singularidades de estos grupos humanos y, por consiguiente, cualquier práctica de comunicación e información que genere procesos de desarrollo ha de privilegiar el respeto y la preservación de la identidad cultural. En cualquier caso, las iniciativas de comunicación alternativa y popular que dan cuenta de las voluntades colectivas locales y nacionales intentan favorecer la transmisión de la realidad indígena, dándoles la posibilidad de que respondan ante las imposiciones de la industria cultural occidental o para que reclamen y protesten contra la injusticia. Se salvaguarda, así, "el derecho a comunicar como un derecho humano fundamental y un requisito de las prácticas verdaderamente democráticas" (González-Pazos, 2000).

Ejemplos más fehacientes son la constitución de redes para la socialización e intercambio de saberes, culturas 
y experiencias a través de la creación de festivales audiovisuales, congresos y talleres locales, nacionales o regionales. Para garantizar que se multipliquen las experiencias de buenas prácticas en comunicación e información para el desarrollo, es necesario un mayor acceso de los pueblos originarios a las TIC; lamentablemente, es un problema no resuelto aún. "El concepto de desarrollo indígena que postulamos depende del reconocimiento y del ejercicio de los derechos de cada uno de los pueblos existentes dentro de cada uno de los Estados" (Ayriwa-Pilco, 2000).

Cabe mencionar las iniciativas emprendidas por el Instituto de Investigaciones de la Amazonía Peruana (IIAP), que desde hace años implementa proyectos y trabaja en la capacitación de líderes y comunicadores indígenas locales. Su más reciente proyecto, Comunidades Indígenas Inteligentes, con la participación del Centro de Alto Rendimiento Computacional, sirvió para crear aplicaciones gratuitas con el objetivo de contribuir a la preservación de las lenguas originarias amazónicas: shawi, asháninka, kukama, ashuar, bora y awajún.

Cuando se habla de comunicación y desarrollo gracias a los medios y a las TIC en pueblos originarios de Latinoamérica, debe reconocerse que estas plataformas favorecen también los procesos de participación social, la formación de valores y normas en la ciudadanía, la divulgación de sus culturas ancestrales, el afianzamiento de la memoria histórica, la capacidad de convocatoria, el diseño y la implementación de estrategias comunicativas apoyadas en la web, además de su impacto en el servicio público local, máxime cuando son los jóvenes indígenas los que tienen mayor acceso a la tecnología, a diferencia de otros grupos etarios.

La tabla 5 muestra la clasificación de tres tipologías de prácticas de comunicación para el desarrollo de los contextos indígenas latinoamericanos y una síntesis de las características que comparten.

Como se refleja en la tabla, estas son algunas modalidades de prácticas de comunicación para el desarrollo; convergen las de carácter institucional $\mathrm{u}$ organizacional, las masivas, las comunitarias y las virtuales, con la presencia de las TIC. Los rasgos expresados son comunes en la mayoría de los pueblos latinoamericanos. Sin embargo, queda claro que de realizarse una aproximación particularizada de cada nación y sus distintos contextos socioculturales indígenas, se encontrarían rasgos específicos, así como el uso de instrumentos y técnicas de comunicación pública propios de sus culturas ancestrales. 


\section{Tabla 5. Prácticas de comunicación para el desarrollo de los contextos indígenas latinoamericanos y sus características comunes}

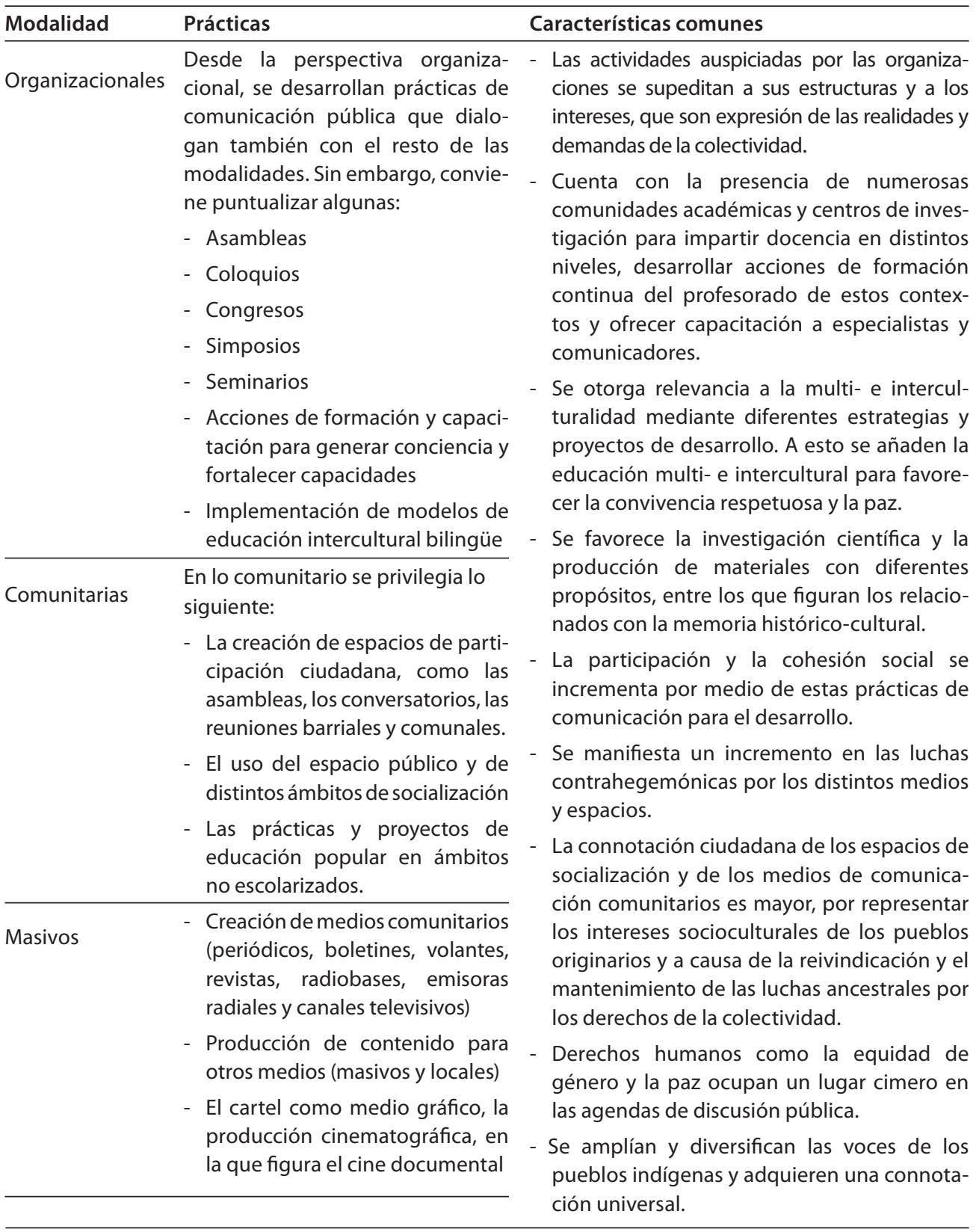


(continuación)

$\begin{array}{ll}\text { Virtuales } & \text { Creación de plataformas digita- } \\ & \text { les, como páginas web y sitios de } \\ & \text { redes sociales } \\ & \text { (Facebook, Twitter, Instagram, } \\ & \text { etcétera) }\end{array}$

Incremento de la producción de contenido y la interacción multicultural gracias a internet y sus plataformas y sitios de redes sociales.

- Defensa de lo ritual y de diferentes códigos que distinguen a cada contexto. Se destacan aquí las creencias, los mitos, las leyendas y cada una de las costumbres y tradiciones que históricamente han formado parte de estas culturas ancestrales.

- Se mantiene la connotación y la dignificación de la naturaleza como parte indispensable tanto de la subsistencia como de la cultura propia.

- La preservación de códigos y representaciones simbólicas cuya significación, tanto cromática como formal y expresiva, constituye un retrato de la identidad cultural de estos contextos.

- Con relación a las prácticas de comunicación que tienen lugar en varias plataformas y en los sitios de redes sociales, fundamentalmente aquellas que se desarrollan con propósitos emancipatorios y de reivindicación por grupos juveniles indígenas, se observan rasgos simbólicos matizados por la reproducción de patrones de la industria cultural occidental, si bien sus propósitos se apegan a su historia y a las realidades y luchas contemporáneas de su contexto.

Elaboración propia

\section{Conclusiones}

Este artículo realizó un breve recorrido por la realidad de los pueblos originarios de Latinoamérica, sus principales necesidades y los problemas que desencadenan corrientes de resistencia y oposición. Se examinaron algunos de los movimientos sociales y populares, los principales centros y organizaciones que han priorizado el diseño y la implementación de proyectos de comunicación para el desarrollo, así como un conjunto de eventos, medios comunitarios y otras tendencias y prácticas de comunicación alternativa que acontecen y que paulatinamente van adquiriendo un lugar importante en el espacio público internacional.

La revisión de las fuentes documentales que se centran en las culturas indígenas de Latinoamérica, en diálogo con los aportes teóricos sobre 
comunicación para el desarrollo, permiten deducir la importancia que reviste hoy la apropiación de nuevos horizontes y categorías analíticas para investigar. Estas no deben restringir las perspectivas tradicionales de estudio, sino abordar otras temáticas, como, por ejemplo, las nuevas prácticas de emancipación, los medios indígenas y sus perspectivas en pleno siglo xxI, el uso de los dispositivos móviles, las ofertas del ciberespacio y las características de los contenidos que se publican, la educomunicación, las plataformas infocomunicativas indígenas, el uso de sitios de redes sociales, la perspectiva de género y los principales espacios, organizaciones, redes, movimientos sociales y eventos que apoyan las causas justas de los pueblos originarios y que contribuyen a la edificación de sus culturas ancestrales.

Todo proyecto encaminado a favorecer el desarrollo de estas culturas tiene que ser compatible con las necesidades objetivas y reales de la colectividad. El objetivo principal será que los actores sean beneficiarios a la vez que participantes en este proceso, teniendo en cuenta elementos claves como el contexto, la flexibilidad y el uso adecuado y eficiente de los medios al alcance. De esta manera, y entendiendo que la cultura debe ser el eje mediador y dinamizador del desarrollo, se conseguirá que estas sociedades se empoderen, que se llegue a la equidad y que se den procesos de racionalización respecto a la opinión que se tiene de estas comunidades. Es aquí donde la comunicación desempeña su rol protagónico, capaz de facilitar y activar cada proceso social y cultural que tenga lugar en estos ámbitos.

Queda claro que las TIC no contribuyen directamente en el desarrollo de los pueblos indígenas. Sin embargo, se trata de un fenómeno global que potencia y amplía el conocimiento acerca de sus demandas económicas, políticas y sociales en el escenario internacional; por tanto, son plataformas de negociación y reafirmación de las identidades culturales y sociales de estos pueblos.

Cualquier estrategia, programa o política de comunicación para el desarrollo en las comunidades indígenas de Latinoamérica ha de concebir a sus habitantes como protagonistas de su propio progreso, o lo que tiende a denominarse autodesarrollo comunitario. En este sentido, se preservan los patrones culturales locales y se evita la imposición de sistemas culturales occidentales, cuyos efectos devastadores son cada vez más evidentes en el continente. Se trata de una comunicación verdaderamente intercultural en la que se privilegian los diálogos respetuosos, de iguales y diversos a la vez, en la que prima la reflexión crítica y constructiva respecto de la búsqueda de soluciones a los problemas contemporáneos. 


\section{Referencias}

ABC Tecnología. (24 de febrero del 2016). Las causas de la "brecha digital" en Latinoamérica. Diario ABC. Recuperado de http://www.abc.es/tecnologia/ moviles/telefonia/abci-causas-brecha-digital-latinoamerica-201602241153_ noticia.html

Agurto, J., y Mescco, J. (mayo del 2012). La comunicación indígena como dinamizadora de la comunicación para el cambio social. Ponencia presentada en el Congreso de la Asociación Internacional de Investigadores de la Comunicación, Montevideo, Uruguay. Recuperado de https://www.servindi.org/pdf/ALAIC_ comunicaci\%C3\%B3nindigena2012.pdf

Alfonso-Murillo, M. (2011). Un tejido de comunicación: medios comunitarios y planes de vida en el norte del Cauca. En M. Pereira y A. Cadavid (Eds.), Comunicación, desarrollo y cambio social: interrelaciones entre comunicación, movimientos ciudadanos y medios (pp. 157-190). Bogotá: Pontificia Universidad Javeriana, Universidad Minuto de Dios y Unesco. Recuperado de http://www.javeriana.edu.co/unesco/ pdf/comunicacion_desarrollo_cambio_social2.pdf

Asociación Pukllasunchis. (s. f). Identificador de la Asociación Pukllasunchis. [logo]. Recuperado de https://www.pukllasunchis.org/

Ayriwa-Pilco, S. (2000). La red de Internet y los Pueblos Indígenas de América Latina: Experiencias y perspectivas (tesis para optar el grado de magíster). Instituto de Estudios de los Medios, Universidad de Bergen. Recuperado de http://www. eurosur.org/TIPI/sami.htm

Banco Mundial. (13 de junio del 2017). Latinoamérica indígena en el siglo xxI. Recuperado de http://www.bancomundial.org/es/region/lac/brief/indigenouslatin-america-in-the-twenty-first-century-brief-report-page

Beltrán, L. R. (febrero de 1993). Comunicación para el desarrollo en Latinoamérica: una evaluación al cabo de cuarenta años. En IV Mesa Redonda sobre Comunicación y Desarrollo. Instituto para América Latina, Lima.

Chaparro-Escudero, M. (2003). El proyecto EMA RTV un reto ante la sociedad de la información y el conocimiento. En La sociedad de la información en el siglo xxi: un requisito para el desarrollo. Buenas prácticas y lecciones aprendidas. España: Ministerio de Ciencia y Tecnología, y ENRED Consultores S. L. Recuperado de https://www. itu.int/net/wsis/stocktaking/docs/activities/1103547250/sociedad-informacionsigloxxi-es.pdf

Chuji-Gualinga, M. (13 de marzo del 2007). Los medios de comunicación indígenas al servicio de los DD. HH. y colectivos. Agencia Latinoamericana de Información [página web]. Recuperado de https://www.alainet.org/es/active/16282 
Consejo Regional Indígena del Cauca. (s. f.). Emisoras Indígenas Filiales [página web]. Recuperado de http://www.cric-colombia.org/portal/red-amcic-emiso ras-indigenas/emisoras-indigenas-filiales/

Coordinadora Latinoamericana de Cine y Comunicación de los Pueblos Indígenas. (s. f.). Observatorio del Derecho a la Comunicación de los Pueblos Indígenas [página web]. Recuperado de http://clacpi.org/observatorio/acerca-de/

González-Pazos, J. (2000). Comunicación para el desarrollo y los pueblos indígenas. Euskonews \& Media 95.zbk (2000 / 10 / 13-20) [página web]. Recuperado de http://www.euskonews.com/0095zbk/gaia9503es.html

Grégor-Barié, C. (2013). La cuestión territorial de los pueblos indígenas en la perspectiva latinoamericana. Recuperado de http://saludpublica.bvsp.org.bo/ textocompleto/bvsp/boxp68/territorio-pueblos-indigenas.pdf

Gumucio-Dagron, A. (2011). Comunicación para el cambio social: clave del desarrollo participativo. Revista Javeriana, 30(58), 26-39. Recuperado de http://revistas. javeriana.edu.co/index.php/signoypensamiento/article/view/2454

Herrera-Miller, K. (2005). Las radios mineras en Bolivia hoy. Mirada diagnóstica a la génesis de la comunicación popular y democrática. Revista Punto Cero, 10(11), 51-58. Recuperado de http://www.scielo.org.bo/scielo.php?script=sci_arttext\& pid=S1815-02762005000200006

Iniciativa de Comunicación para el Desarrollo Sostenible. (2010). Prácticas de CpD para el cambio climático en América Latina. Iniciativas, actores, metodologías y resultados. En La comunicación para el desarrollo ante los desafíos del cambio climático, manejo de recursos naturales, gestión del riesgo y seguridad alimentaria. Recuperado de http://www.fao.org/3/a-k7361s.pdf

Iniciativa de Comunicación para el Desarrollo Sostenible. (2011). Comunicación y Desarrollo de los Pueblos Indígenas de América Latina: Experiencias y retos. En La Comunicación en la Gestión Territorial, el Cambio Climático y el Desarrollo de los Pueblos Indígenas [documento técnico]. Recuperado de http://www.fao. org/3/a-ap608s.pdf

MacDonald, T. (2003). Un enfoque de los derechos indígenas a comienzos del siglo xxI: Normas internacionales, movimientos sociales y reclamos de ciudadanía. Recuperado dehttps:/ scholar.harvard.edu/tmacdon/files/Macdonald_HumanRights21stCentury_ Spanish.pdf

Mena, D., y Álvarez, A. (2015). Proyecto Caracol: Alternativa de comunicación para el desarrollo local en el municipio Cumanayagua. Redes.com, (11). Recuperado de http://revista-redes.hospedagemdesites.ws/index.php/revista-redes/article/ view/332 
Paiba-Samamé, J. A. (22 de febrero del 2013). La brecha digital en América Latina [página web]. Recuperado de http://www.redem.org/la-brecha-digital-en -america-latina/

La Iniciativa de Red de Comunicación. (12 de febrero del 2009). Plataforma de Comunicación y Desarrollo de los Pueblos Indígenas de América Latina [página web]. Recuperado de http://www.comminit.com/la/content/ plataforma-de-comunicaci\%C3\%B3n-y-desarrollo-de-los-pueblosind $\%$ C3\%ADgenas-de-am\%C3\%A9rica-latina

Programa de las Naciones Unidas para el Desarrollo. (14 de junio del 2016). Informe regional sobre desarrollo humano para América Latina y el Caribe 2016. Recuperado de http://www.latinamerica.undp.org/content/rblac/es/home/ library/human_development/informe-regional-sobre-desarrollo-humanopara-america-latina-y-e.html

Ponce de León, R. (2011). Pueblos originarios, estigmatización e invisibilización en los medios audiovisuales argentinos. Escritos en la Facultad, 14(69). Recuperado de http://fido.palermo.edu/servicios_dyc/publicacionesdc/vista/detalle_articulo. php?id_libro=336\&id_articulo $=7543$

Rodríguez-Mir, J. (2008). Los movimientos indígenas en América Latina. Resistencias y alteridades en un mundo globalizado. Gazeta de Antropología, 24(2). Recuperado de http://hdl.handle.net/10481/6928

Stavenhagen, R. (1999). Structural racism and trends in the global economy. Int. Counc. Hum. Rights Policy, (25), 1-17.

Unicef. (2017). Los pueblos indígenas en América Latina. Recuperado de https://www. unicef.org/lac/pueblos_indigenas.pdf

Vargas-Hernández, J. (2005). Movimientos sociales para el reconocimiento de los movimientos indígenas y la ecología política indígena. $\mathrm{Ra}$ Ximhai, 1(3), 453-470. Recuperado de http://www.uaim.edu.mx/webraximhai/ Ej-03articulosPDF/02\%20mov\%20sociales.pdf

Zambrano-Cabanilla, E. (17 de abril del 2006). El legado de los pueblos indígenas. Agencia Latinoamericana de Información [página web]. Recuperado de https:// www.alainet.org/es/active/11176

8300 web. (6 de diciembre del 2010). La invisibilización de los pueblos originarios. [página web]. Recuperado de http://www.8300.com.ar/2010/12/06/la-invisibi lizacion-de-los-pueblos-originarios/ 